\title{
EDITORIAL
}

\section{Diffuse large B-cell lymphoma: is salvage possible after failure of second-line treatment?}

\author{
Bone Marrow Transplantation (2016) 51, 1; doi:10.1038/ \\ bmt.2015.225; published online 5 October 2015
}

Since the publication of the PARMA study, ${ }^{1}$ second-line salvage therapy followed by high-dose chemotherapy and autologous stem cell transplantation (ASCT) became and continues to be the standard of care for chemosensitive relapsed diffuse large B-cell lymphoma (DLBCL), even in the rituximab era. However, prospective randomized trials, such as the Collaborative trial in relapsed aggressive lymphoma (CORAL), ${ }^{2}$ showed that salvage therapies for DLBCL are not always effective and that only half of the patients will proceed to ASCT. This is mainly because of an insufficient response to second-line treatment. Little is known about those who fail second-line therapy.

In this issue, Van Den Neste et $a l^{3}{ }^{3}$ report the outcome of 203 patients who could not proceed to scheduled ASCT in the CORAL study, mostly due to treatment failure (170 patients) defined as stable disease or progressive disease after second-line therapy (rituximab-carboplatin-etoposide (RICE) or rituximab-dexamethasone-cytarabine-cisplatin (R-DHAP) depending on the randomization arm). This is a particularly challenging population and the study by Van Den Neste et al. represents by far the largest in such a setting. Median overall survival (OS) for the entire population was 4.4 months and the 1 -year OS was $23 \%$, hence the outcome after failure of second-line treatment in DLBCL is poor, as expected.

The main take home message from this study is that objective responses could still be achieved after third line by switching to combination chemotherapy or chemoimmunotherapy that are not sharing cross-resistance with prior therapy(ies). Patients who initially received RICE or R-DHAP regimen were shifted to DHAPlike or ifosfamide-carboplatin-etoposide (ICE)-like respectively, or received other regimens. Overall response rates to third-line chemotherapy were $39 \%$, including $27 \%$ complete response (CR), and no third-line regimen was significantly superior to the others. Importantly, patients amenable to transplantation after third-line chemotherapy achieved a significantly prolonged survival with a median OS of 11.1 months in 64 patients (31.5\%) who were eventually transplanted, mostly with ASCT $(n=56)$, compared with 3.3 months in those who were not. Accordingly, 2-year OS were 33.9 and $9.3 \%$, in transplanted versus non-transplanted patients, respectively. An encouraging, yet somehow unexpected result, was that median OS was not reached in patients achieving a $\mathrm{CR}$ after a third-line regimen and autografted afterwards (1-year OS of $88.4 \%)$.

Based on this study, offering third-line chemotherapy with an intent to achieve an objective response (preferably $C R$ ) and proceed with ASCT ought to be considered the standard approach in relapsed DLBCL when second-line treatment fails. However, there is an unmet need to improve response rates; accordingly, results from this study shall constitute the control arm against which new drug combinations including novel candidate targeted therapies ought to be tested.

\section{CONFLICT OF INTEREST}

The author declares no conflict of interest.

\author{
A Bazarbachi \\ Department of Internal Medicine, American University of Beirut, \\ Medical Center, Beirut, Lebanon \\ E-mail: bazarbac@aub.edu.lb
}

\section{REFERENCES}

1 Philip T, Guglielmi C, Hagenbeek A, Somers R, Van der Lelie H, Bron D et al. Autologous bone marrow transplantation as compared with salvage chemotherapy in relapses of chemotherapy sensitive non-Hodgkin's lymphoma. N Engl J Med 1995; 333: 1540-1545.

2 Gisselbrecht C, Schmitz N, Mounier N, Singh Gill D, Linch DC, Trneny M et al. Rituximab maintenance therapy after autologous stem-cell transplantation in patients with relapsed CD20(+) diffuse large B-cell lymphoma: final analysis of the collaborative trial in relapsed aggressive lymphoma. J Clin Oncol 2012; 30: 4462-4469.

3 Van Den Neste E, Schmitz N, Mounier N, Gill D, Linch D, Trneny M et al. Outcome of patients with relapsed diffuse large B-cell lymphoma who fail second-line salvage regimens in the international CORAL study. Bone Marrow Transplant 2016; 51: 51-57. 